Recent Insights into the Physics of the Sun and Heliosphere:

Highlights from SOHO and Other Space Missions

IAU Symposium, Vol. 203, 2001

P. Brekke, B. Fleck, and J. B. Gurman eds.

\title{
Magnetic Field Reconstruction in a Solar Active Region
}

\author{
H. Wang and Y. Yan \\ National Astronomical Observatories, Chinese Academy of Sciences, \\ Beijing 100080, China \\ T. Sakurai \\ National Astronomical Observatory of Japan, 2-21-1 Osawa Mitaka, \\ Tokyo 181-8588, Japan
}

\begin{abstract}
Supposing coronal magnetic fields are in a force-free state from the chromosphere to the height of two solar radii, we reconstruct 3D force-free magnetic fields by making use of a new numerical technique, in which the fields are represented by a boundary integral equation based on a specific Green's function. Vector magnetic fields observed on the photospheric surface can be taken as the boundary conditions of this equation. Magnetic fields in AR8270 on 14 July 1998 were employed as an example to exhibit the capability of this numerical technique.
\end{abstract}

\section{Introduction}

The solar magnetic field plays an important role in the solar atmosphere. It is widely believed that coronal structures delineate magnetic field lines anchored on the photospheric surface. Since magnetic fields on the photospheric surface can be well measured, a reconstruction of the 3-D solar magnetic field above this surface is necessary to explore structures of coronal magnetic fields. Several techniques for this reconstruction have been reviewed by Sakurai( 1989) McClymont et al (1997) Yan and Sakurai (1997). We here employ a new numerical method suggested by Yan and Sakurai (2000).

\section{Numerical computation}

The 3-D force-free magnetic fields in AR8270 on 14 July 1998 were computed by means of a new numerical technique (Yan and Sakurai, 2000) in which forcefree fields are represented by a boundary integral equation based on a specific Green's function. The photospheric vector magnetic fields measured in AR8270 with the Multiple Channel Solar Telescope at Huairou Solar Observing Station in Beijing were taken as the boundary conditions of this equation.

A package of IDL software suitable for large data arrays was developed, such software being able to draw 800 magnetic field lines on a boundary surface with $150 \times 150$ grid points in only 40 minutes by means of a PentiumIII600 
personal computer. This package, as is clear, was useful to deal with a large amount of observational data.

In this numerical extrapolation, the following two points were emphasized: (1) The reconstructed 3-D fields satisfied the divergence-free and force-free conditions with high precision (Wang, Yan and Sakurai, 2000). (2) A new method

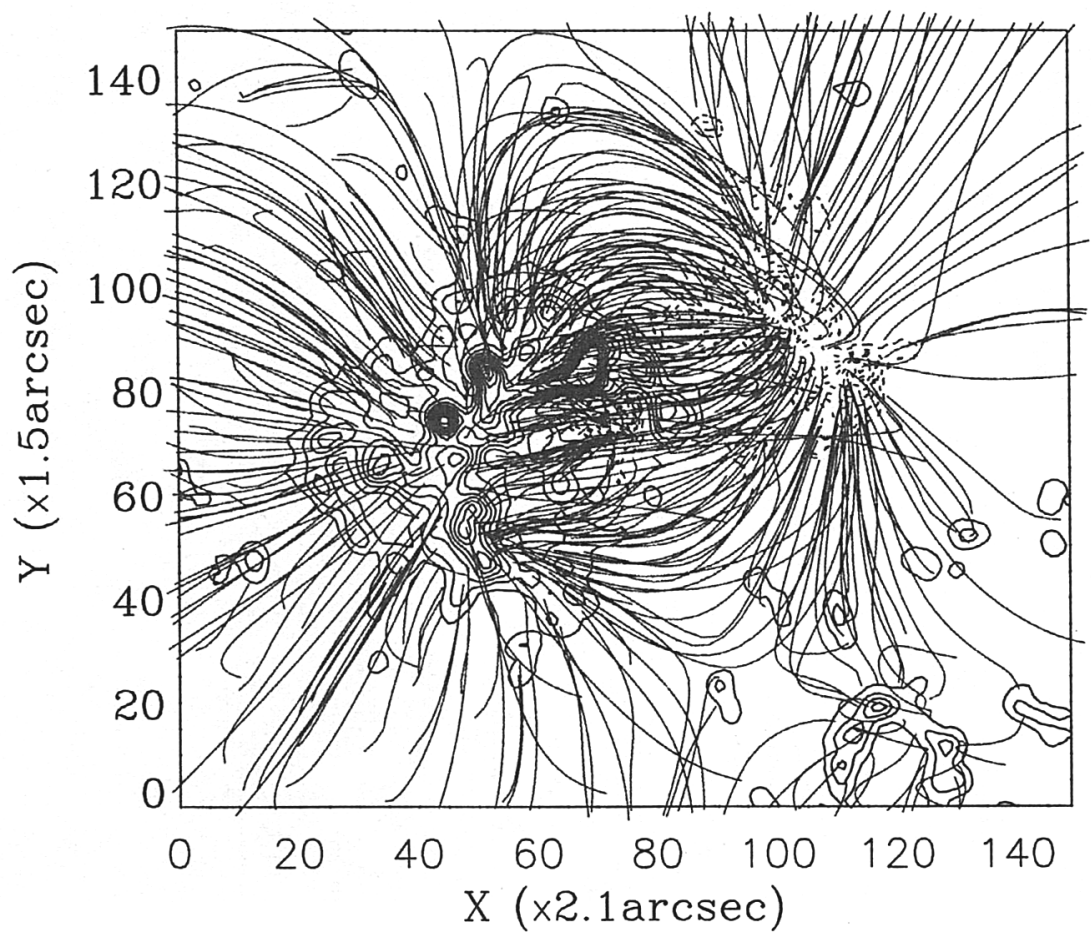

Figure 1. The reconstructed 3-D magnetic fields in AR8270 at 09:19UT of 14 July 1998

for data reduction was proposed (Wang, Yan, and Sakurai, 2000), which making reconstructed fields be in agreement with structures in TRACE images.

\section{Conclusion}

The following conclusions can be drawn from our results: (a) The boundary integral method is a promising method in numerical computations of a 3-D static solar magnetic field. (b) Structures in the reconstructed 3-D magnetic fields are in agreement with those in TRACE images. This fact means that the boundary integral equation describes coronal magnetic fields very well. (c) The reduction of boundary data is very important for the reconstruction of 3-D coronal magnetic fields due to uncertainties in these data. Our new method for data reduction makes the results of numerical computation reliable. (d)Since the 
1998-Jul-14

$12: 05: 41$

$d t=0.0$

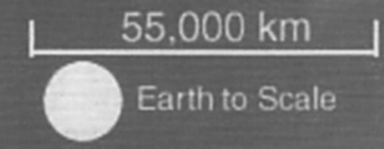

Figure 2. An EUV image of AR8270 observed by TRACE

boundary data are calibrated with a linear force-free field model, the computed $3 \mathrm{D}$ magnetic filed can be regarded as a quasi-linear force-free field approximation.

\section{References}

Fletcher, C. 1984, Computational Galerkin Methods, Springer- Verlag, Tokyo. McClymont A. N., Jiao, L. and Mikic Z. 1997 Solar Physics, 174, 191 Sakurai, T. 1989, Space Sci. Rev., 512, 11 Yan, Y. and Sakurai, T. 1997, Solar Physics, 174, 65 Yan, Y. and Sakurai, T. 2000, Solar Physics, in press Wang, H. N., Sakurai, T. and Yan, Y. 2000, Solar Physics, in press 